\title{
Compliant mechanisms for an active cardiac stabilizer: lessons and new requirements in the design of a novel surgical tool
}

\author{
L. Rubbert ${ }^{1}$, P. Renaud ${ }^{1}$, W. Bachta ${ }^{2}$, and J. Gangloff ${ }^{1}$ \\ ${ }^{1}$ LSIIT, Université de Strasbourg-CNRS, Strasbourg, France \\ ${ }^{2}$ ISIR, Université Pierre et Marie Curie-CNRS, Paris, France
}

Received: 24 February 2011 - Revised: 12 May 2011 - Accepted: 26 May 2011 - Published: 14 June 2011

\begin{abstract}
In this paper, three aspects of the use of compliant mechanisms for a new surgical tool, an active cardiac stabilizer, are outlined. First, the interest of compliant mechanisms in the design of the stabilizer is demonstrated with in vivo experimental evaluation of the efficiency of a prototype. We then show that the specific surgical constraints lead to the development of compliant mechanisms, with the design of new original mechanical amplifiers. Finally, the requirements in the design of stabilizers exhibiting a higher level of integration are outlined. Novel architectures and design procedures are actually needed, and we introduce an exploratory study with a proof-of-concept designed using the combination of ant colony optimization and classical pseudo rigid body modeling. Relative errors in the estimation of the displacement do not exceed $5 \%$. The proposed design method constitutes an interesting approach that may be applied more generally to the design of compliant mechanisms.
\end{abstract}

\section{Introduction}

Compliant mechanisms exhibit higher accuracy and compactness than conventional mechanisms. They can consequently contribute to the development of Minimally Invasive Surgery (MIS) procedures, high accuracy procedures that require small size instruments. The use of compliant devices also simplifies the sterilization process (Rebello, 2004), and the suppression of lubrication improves surgical compatibility. Surgical tools for organ manipulation (Awtar et al., 2010), grasping (Frecker et al., 2005a) or tissue cutting (Frecker et al., 2005b) have therefore been proposed previously.

In the Cardiolock project, we investigate the design of an active cardiac stabilizer, a new surgical tool for heart surgery. In this context, this article has three aims. First, we show that the field of heart surgery can benefit from the field of compliant mechanisms by outlining the efficiency of a compliantbased active cardiac stabilizer with in vivo experimental re- sults. Second, we show that the surgical context introduces design requirements that can lead to contributions in the field of compliant mechanisms. An original compliant mechanism is introduced, whose architecture is indeed directly derived from the surgical necessities. Finally, we propose an exploratory study on the design of a highly integrated active stabilizer: a proof-of-concept is introduced with a design methodology based on ant colony optimization and pseudo rigid body modeling.

The paper is organized as follows. The principle of active stabilization and the efficiency of the use of a compliant mechanism for active stabilization are presented in Sect. 2. The design of an original compliant mechanism based on the surgical requirements is introduced in Sect. 3, with the use of kinematic singularities to synthesize an amplification mechanism. Then, in Sect. 4, a design methodology is proposed to assess the level of integration that can be achieved in order to design an active stabilizer. A very recent proof-of-concept is experimentally evaluated, before concluding in Sect. 5 . 


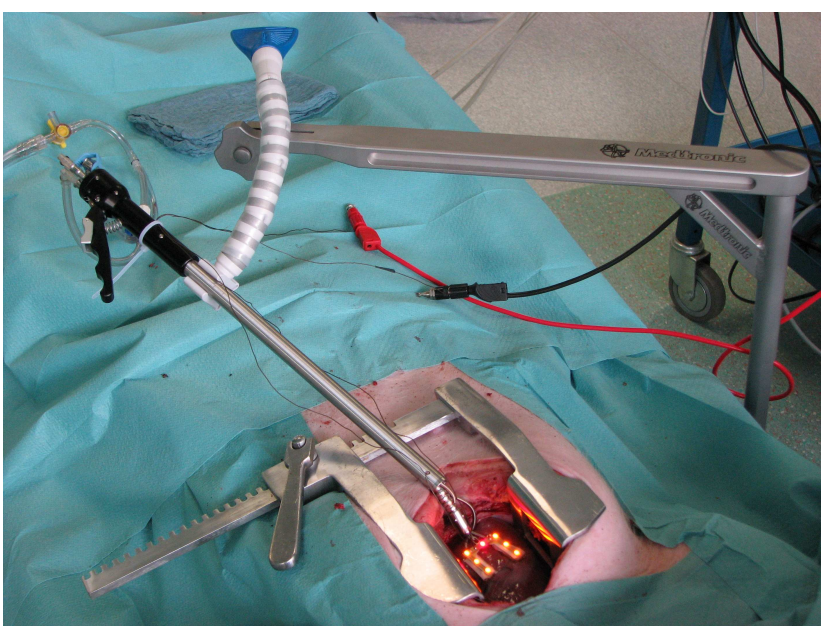

Figure 1. Passive stabilizer during in vivo evaluation on pig.

\section{Active stabilization for cardiac surgery}

In the field of heart surgery, one of the most common interventions is Coronary Artery Bypass Grafting (CABG). In order to improve the supply of blood to the myocardium, arterial or vein grafts are connected to the coronary arteries. The most delicate part of a CABG is the suture of the grafts to the coronary arteries. These arteries have a diameter of 1-2 mm, and more than 10 knots must be performed around each artery. For the patient, the best approach is to perform such a procedure on a beating heart, i.e. without the use of an external heart-lung machine, and with a minimally invasive approach. Only small incisions are then made to insert the surgical tools and an endoscope through trocars.

Today, in that situation surgeons use a stabilizer, a device that aims at locally immobilizing the beating heart surface during the suturing process. In a MIS context, this stabilizer is composed of fingers applied on the heart and a long shaft (Fig. 1) that is inserted through the subxiphoid process, at the base of the sternum, to reach the area of interest on the heart surface.

From a mechanical point of view, one can easily understand that the forces developed by the heart, in the order of $5 \mathrm{~N}$ (Bachta et al., 2008), cause the deformation of the mechanism. The current commercial device (Fig. 1) exhibits displacements at its tip that exceed the required accuracy, as outlined by surgeons (Cattin et al., 2004; Lemma et al., 2005). Displacements of the stabilizer tip should remain in the order of $0.1 \mathrm{~mm}$, with respect to the suturing task described earlier.

As a consequence, we have proposed in the Cardiolock project (Bachta et al., 2007) the development of an active device to improve the stabilization accuracy and to allow CABG to be performed satisfactorily on a beating heart with MIS. The main idea is to use the endoscope introduced during the surgery (Fig. 2) to observe the presence of any deflection of the stabilizer and compensate, in real time, for the

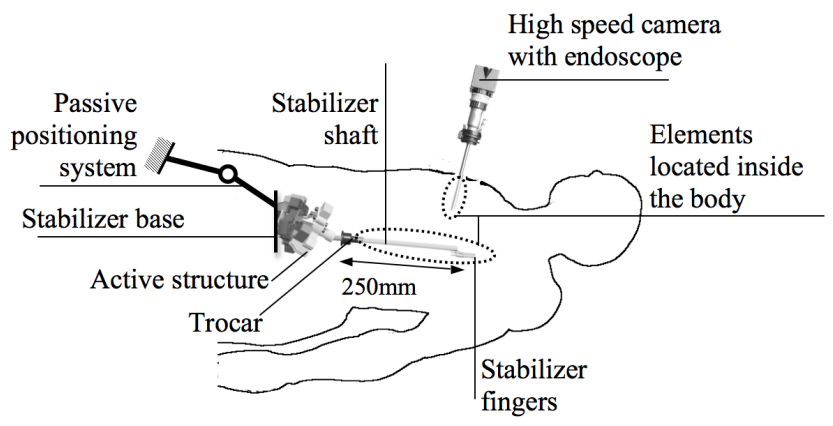

Figure 2. Principle of insertion of the active stabilizer.

displacement of the area of interest. Compliant mechanisms obviously appear of interest when combined with piezoelectric actuation: high dynamics can be obtained without any backlash. Thus the Cardiolock principle relies on the use of a compliant mechanism controlled by a piezoelectric actuator using the information given by the endoscope.

\section{Compliant mechanisms for a new surgical tool}

\subsection{Design requirements}

The stabilization task consists of compensating, in the presence of the heart force, for displacements in the order of $1 \mathrm{~mm}$ (Bachta et al., 2008). The main source of displacement of the stabilizer tip is the bending of the shaft. As a consequence, two directions of compensation at the stabilizer tip are needed. The displacement along the shaft axis is not significant. Forces exerted by the heart on the stabilizer have been assessed experimentally (Bachta et al., 2008). The amplitude of the force is in the order of $5 \mathrm{~N}$ in the direction perpendicular to the stabilizer shaft.

The stabilizer is in interaction with the heart at its tip, but also with the trocar that allows its insertion into the patient's body. As a consequence, the movement of the trocar due to respiratory motion creates forces on the stabilizer body in addition to the heart forces. A simple way to avoid these additional perturbations increasing the complexity of the stabilizer control is to consider that the compliant mechanism and the actuator are located outside the body and allow the stabilizer shaft to rotate with respect to the trocar. This simultaneously simplifies the sterilization of the device, since the active elements are located outside the patient's body. Revolute joints cannot be located at the trocar position, which means that the stabilizer must exhibit a Remote Center of Motion (RCM).

\subsection{Design of a first device: Cardiolock 1}

Cardiolock 1 is a first prototype that has been designed to assess the efficiency of the active stabilization approach during in vivo experiments. Experiments on pigs cannot be 


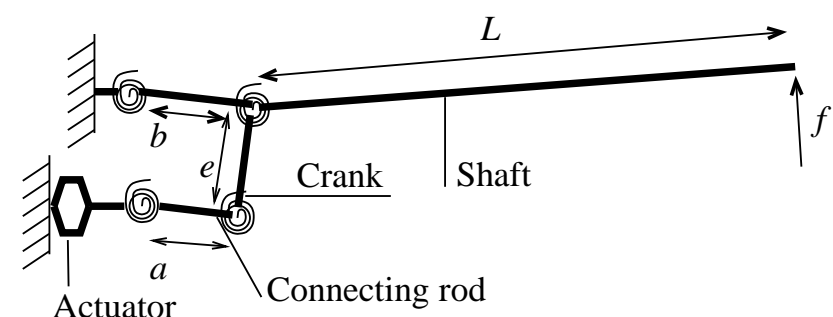

Figure 3. PRBM model of the device.

performed with a MIS approach for anatomical reasons. The RCM constraint was therefore not taken into account and compensation is performed only in one direction.

An actuator with integrated amplification structure (Cedrat Technologies APA120ML) is considered. Its translational movement is converted using a slider-crank system (Fig. 3). The stabilizer shaft is connected to the crank, the actuator is the slider which is connected to the crank by means of a connecting rod. The mechanism synthesis has been carried out using a Pseudo Rigid Body Model (PRBM, Howell, 2001). A torsion spring is used to represent each compliant joint and an additional joint is introduced to represent the flexibility of the shaft. The achievable displacement can be easily derived from Fig. 3 (Bachta et al., 2007). The stiffness of each compliant joint is described with the analytical model of symmetric right-circular profile joints (Howell, 2001) and the model from Pham and Chen (2002) gives the maximum stresses in the joints. The parameters to be determined are the geometrical parameters $(a, b, e)$ and the minimum thickness $t$ of the compliant joints. A non linear optimization was achieved to determine the best set of parameters. The optimization criterion is the size of the device. The compensation condition as well as the maximum admissible stresses in the joints are considered as non linear constraints. The CAD view of the system corresponding to the result of the optimization is introduced in Fig. 4. Further evaluation of the PRBM accuracy by comparison with simulations using Finite Element Analysis (FEA) showed the relevance of the model. The stabilizer tip maximum displacement is correctly described by the PRBM as well as its stiffness.

\subsection{Experimentation - stabilization efficiency}

The stabilizer tip displacement given by the PRBM could be reached within $5 \%$ with laboratory experiments. The device stiffness and the eigenfrequencies are however significantly different from the values estimated with FEA: eigenfrequencies are for instance lowered by more than $13 \%$. Further analysis demonstrated that the assembly of the mechanism, and especially the connection of the connecting rod to the actuator lowers the device's performances. Even though the elements are rigidly connected, the behavior of the device remains very sensitive to the quality of assembly.

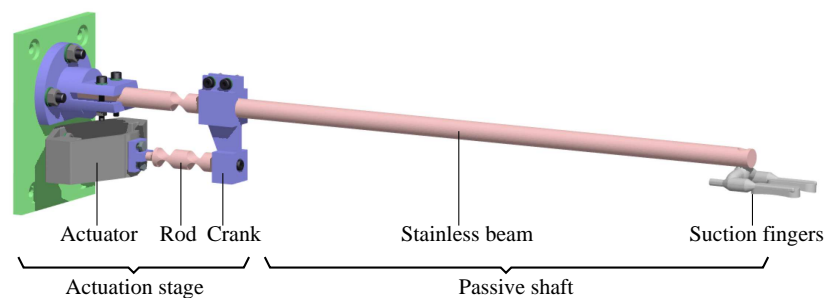

Figure 4. CAD view of Cardiolock 1, an active stabilizer with 1 DOF.
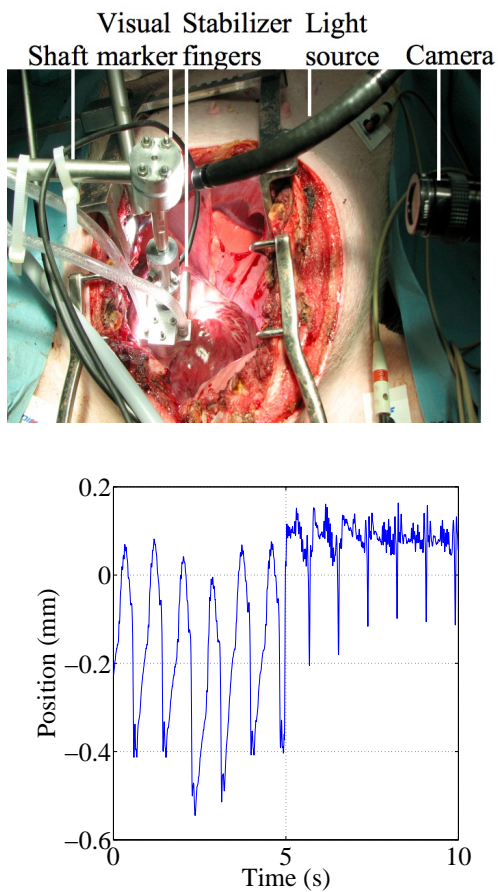

Figure 5. Cardiolock 1 prototype during in vivo experiments (left) and corresponding recorded residual displacement (right).

After the implementation of the stabilizer control, in vivo experiments have been performed. The stabilizer tip is positioned above the sternum of a $35 \mathrm{~kg}$ pig, with a custom end-effector to reach the beating heart. A high-speed camera (Dalsa CAD-6, 333fps) is used to detect the stabilizer tip displacement. It is positioned in front of the stabilizer tip to follow a marker attached to the stabilizer tip. Because of the anatomy of the animal, a custom end-effector is added to access to the heart surface.

The position of the stabilizer tip measured with the camera is shown in Fig. 5. During the active stabilization, after $6 \mathrm{~s}$ on the graph, the standard deviation of the position of the stabilizer tip is equal to 35 microns. The device efficiency is high enough to confirm the benefit of using a compliant mechanism in combination with vision to develop a new surgical tool, an active stabilizer. 


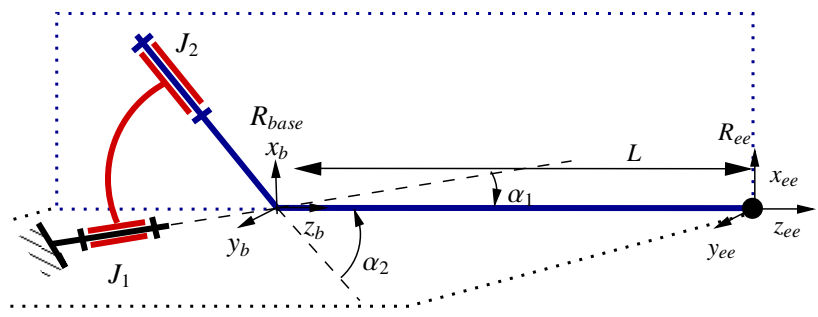

Figure 6. Kinematic scheme of Cardiolock 2.

\section{A new compliant device from surgical requirements: Cardiolock 2}

\subsection{Stabilizer kinematics}

As introduced in Sect. 3.1, the design objective is to provide the stabilizer with $2 \mathrm{DOF}$ in rotation, with respect to the RCM located at the trocar. Parallel architectures can be considered, with spatial arrangements (Gosselin and Angeles, 1989; Gregorio, 2004), sometimes with the use of spherical joints, but they seem very difficult to manufacture as compliant devices. The experimental evaluation of Cardiolock 1 has also shown that the complexity of the design has to be limited, in particular with a small number of elements in the assembly to minimize sources of flexibility. As a consequence, a simple serial mechanism is considered as represented in Fig. 6 (Bachta et al., 2009). Displacements around the represented configuration can be obtained by the rotation of joints $J_{1}$ and $J_{2}$, with a decoupling of the two displacements that simplifies the stabilizer control.

The displacement of the stabilizer tip is a function of the angles $\alpha_{1}$ and $\alpha_{2}$, the length $L$ being constrained by the medical requirements. From a dynamic point of view, parameters $\alpha_{1}$ and $\alpha_{2}$ should be minimized to get a compact structure with lower inertias. This implies the development of compliant joints actuated with piezoelectric actuators that provide large rotations.

\subsection{From kinematic singularities to mechanical amplifiers}

Piezoelectric actuators provide linear motions that need to be converted into rotations, that we want to maximize. Parallel mechanisms can exhibit a behavior that corresponds to that situation, in the vicinity of the so-called parallel singularities (Gosselin and Angeles, 1990). Even though such configurations are rarely considered (Stoughton and Arai, 1992; Ranganath et al., 2006), we have thus proposed (Bachta et al., 2009) consideration of a parallel mechanism to design the compliant mechanism that would convert the actuator displacement into a rotation. The parallel mechanism at the origin of our amplifier is represented in Fig. 7. Parallel singularity corresponds to the situation $\epsilon=0$.

Considering the points $A_{1}$ and $A_{3}$ as immobilized and an actuator is driving the point $A_{2}$, the mechanism becomes

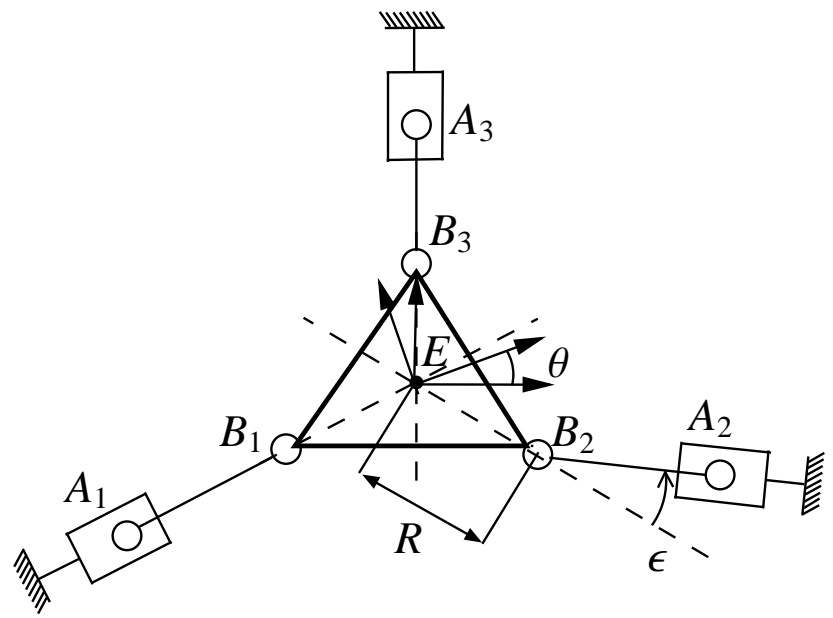

Figure 7. 3PRR parallel mechanism close to singularity.

equivalent in the represented configuration to a revolute joint, whose rotation amplitude is simply related to the actuated displacement $\delta q$ of point $A_{2}$ :

$\delta \theta=\frac{1}{R \sin (\epsilon)} \delta q$

with $R=\left\|E B_{2}\right\|$. We can easily set the amplitude of the rotation by modifying the value of $\epsilon$, and obtain a high rotation/translation ratio. The obtained mechanical amplifier is also interesting because of its stiffness properties. In-plane stiffness is high due to its parallel nature, and out-of-plane stiffness is easily controlled by the width of the mechanism.

\subsection{Design and experimentation}

Selection of the device's geometrical parameters has been achieved using an iterative design process, analyzing the performances of the device with FEA. The prototype, represented in Fig. 8, exhibits displacements at the tip of the stabilizer shaft equal to $1.28 \mathrm{~mm} \times 1.28 \mathrm{~mm}$, which is consistent with the results of FEA and correspond to the design requirements.

\section{What are the limits of the integration?}

To deal with the interaction of the stabilizer with the trocar, RCM architecture has first been considered. Even if the device efficiency is satisfactory, its size still limits its ease of use. More importantly, placing the actuators outside the body simplifies the sterilization process but greatly increases the complexity of the control, because of the non-collocated sensor and actuator configuration. New requirements in the design of an active stabilizer appear: we need compliant architectures that allow the integration of actuators inside the stabilizer shaft, close to the stabilizer tip, and we need to 


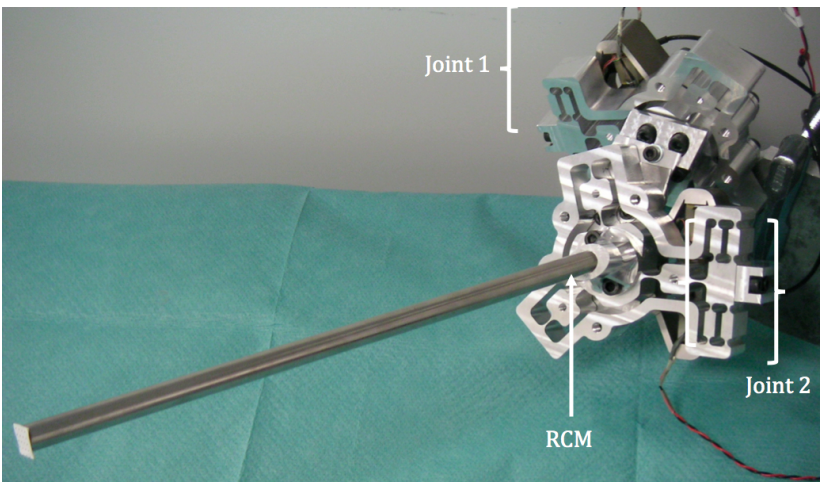

Figure 8. The Cardiolock 2 prototype.

identify methodologies for their design. In this paper, ongoing research on the design of a fully integrated device is presented with a proof-of-concept whose design is obtained using ant colony optimization associated with pseudo rigid body modeling.

\subsection{Design methodology and architecture selection}

\subsubsection{Design methodology}

As a first step, we consider the problem of the integration of a compliant mechanism and its piezoelectric actuator for a 1 DOF mechanism. Because of the strong size constraints, the actuator is considered to be positioned along the shaft axis, providing an axial displacement. A piezoelectric actuator that can be integrated in the shaft presents maximum displacements in the order of 50 microns. A compliant mechanism has therefore to be designed to first convert the displacement along the shaft axis into a displacement perpendicular to the shaft, and second to amplify this displacement. The stabilizer tip displacement amplitude should be in the order of $1 \mathrm{~mm}$.

The synthesis of the mechanism can be made by a topology optimization or by selection and optimization of a predefined architecture. 1 DOF amplification mechanisms have been widely studied, and we thus elaborate a transformation mechanism from existing solutions before performing its optimization.

\subsubsection{Mechanism selection}

A review of the existing transformation mechanisms has been performed. Two mechanisms are of particular interest: the Scott-Russell mechanism (Tian et al., 2009) and the four-bar mechanism (Parkinson et al., 2001). The first one transforms a translation movement into another translation movement in the perpendicular direction and its oblong shape tends to be compatible with an integration inside the stabilizer shaft. The second one can amplify rotations (Sitti, 2003) as well as translations (Choi et al., 2010; Liaw and Shirinzadeh, 2008).

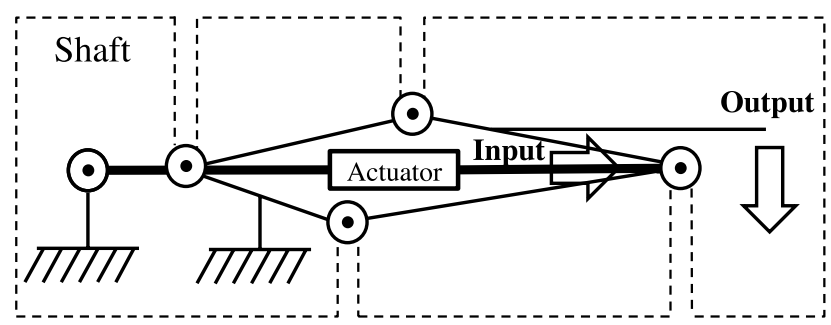

(a) Kinematic scheme of the proposed 4-bar mechanism. The dotted lines represent the contour of the bars in the structure.

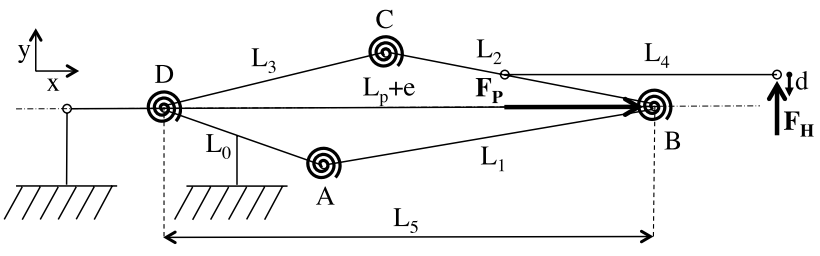

(b) Parameterization of the PRBM of the mechanism.

Figure 9. Description of the proposed active stabilizer with embedded actuation.

The compliant mechanism has to be manufactured from the stabilizer shaft, of cylindrical shape. In this context, the elements that compose the Scott-Russell mechanism have slender shapes and may lack stiffness. The four-bar mechanism is therefore chosen (Fig. 9a), with the integration of the piezoelectric actuator inside a tubular shaft. With this configuration, the force delivered by the actuator introduces tensile stresses in the mechanism bars, without any risk of buckling. To obtain the corresponding compliant mechanism, circular notch joints are preferred to leaf spring joints for their ease of manufacture and their better accuracy (Trease et al., 2004).

\subsection{Optimization with Ant colony algorithm}

\subsubsection{Modeling}

The use of a PRBM appeared in the design of Cardiolock 1 as an efficient way to describe the mechanism during its optimization. This approach is therefore still considered for this new device. The model is represented in Fig. 9b. Six geometrical parameters define the mechanism and its initial configuration $\left(L_{0}, L_{1}, L_{2}, L_{3}, L_{4}\right.$ and $\left.L_{5}\right)$. Since the mechanism is integrated in a tube, its outer radius $R$ and its thickness $T$ are also needed to define the geometry. The compliant joints are described by their minimum thickness $t$, the radius $r$ of their circular profile and their width, defined by their position with respect to the tube axis.

The relationship between the actuator displacement $e$ and the output displacement $d$ can be easily obtained by expressing loop closure equations. To obtain the achievable output displacement, a static model is derived, since the piezoelectric actuator maximum displacement depends on the stiffness 
of the mechanism acting against it. The compliant joint rotational stiffnesses are described using the model derived by Schotborgh et al. (2005), described as the most accurate and having the widest range of validity by Yong et al. (2008).

The stresses in the compliant joints can be expressed analytically. Stress concentration factors are included to improve the accuracy (Pilkey and Pilkey, 2008).

\subsubsection{Ant Colony Optimization for compliant mechanisms}

The compliant architecture is defined by sixteen geometrical parameters: six bar lengths, four joint thicknesses, four radii circular notches, one tube diameter and one tube thickness. During the optimization, we consider that the actuator is given, since we will use the actuator providing the maximum displacement that can be integrated in the tube, and the tube material properties are known. The objective of the optimization is then to maximize the output displacement $d$, while satisfying a set of constraints:

- The maximum stresses in the joint must be compatible with the material properties.

- The mechanism profile must remain inside the tube's outer shape.

- The angles between the mechanism bars must remain compatible with the configuration represented in Fig. 9 b.

- The geometry of the joints must be compatible with the shape of the bars as represented in Fig. 9a.

- The model developed in Schotborgh et al. (2005) must remain valid, which includes the existence of a symmetry of the compliant joints with respect to their neutral axis.

- The actuator must fit in the tube.

This constitutes a set of seven linear constraints and fifteen non-linear constraints represented as a set of inequalities. For such a highly constrained problem, and a large number of parameters, gradient-based optimization algorithms usually have a low efficiency, particularly with the presence of many local minima in the optimization function. Metaheuristic optimizations, based on stochastic algorithms, can constitute interesting alternatives. Genetic algorithms and evolutionary algorithms are well known approaches. Hereafter, we propose to investigate the use of Ant Colony Optimization (ACO) in our context. ACO is essentially interesting for its ease of implementation because the method does not need a delicate tuning of many internal parameters to be efficient.

ACO mimics the behavior of ants that are able collectively to optimize the path between their nest and a source of food. ACO is therefore performed by randomly generating sets of solutions, and iteratively restraining the generation of solutions around the most interesting solutions found in a previous step. Considered initially for combinatorial problems (Dorigo and Gambardella, 1997), it has been extended to continuous domain (Blum, 2005; Socha and Dorigo, 2008), which is our case. ACO has been proposed for structural topology optimization (Kaveh et al., 2008; Luh and Lin, 2009) but, to the authors knowledge, not yet considered for the optimization of a compliant mechanism.

Before introducing the adaptation of ACO to our context, we need to define a performance function to distinguish and rank possible solutions. The displacement $d$ has to be maximized. For a given material, the objective is also to use in an optimal way its mechanical properties. In other words, it can be interesting to introduce into the performance function the closeness of the maximum stress to the admissible maximum stress for the material. The proposed performance function is expressed as:

Performance $(\xi)=\frac{d}{1.01^{\text {abs }\left(\sigma_{\text {objective }}-\sigma_{\max }\right)}}$

with $\xi$ the parameter set, $\sigma_{\text {objective }}$ the maximal admissible stress, $\sigma_{\max }$ the maximum stress in the mechanism and 1.01 a penalty factor chosen for the problem. Since ACO does not need any continuity or derivation property for the performance evaluation function, such a non-linear function can be considered and indeed will efficiently rank the solutions since the stress value is "locked" to the admissible value.

Adapted to our context, the algorithm is composed of four main steps (Fig. 10). In the first step, sets of geometrical parameters are generated randomly, using a uniform distribution law in all the parameter domain. To increase the chance of finding a viable mechanism, the constraints are divided into two sets of different importance. The most important constraints are initially considered before working in a parameter domain around the identified solutions to determine parameter sets that respects the whole set of constraints.

In the second step, for each parameter set that respects all the constraints, a new set of parameters is generated in a restrained domain surrounding the solution to improve the solution performance. The restrained domain corresponds to $20 \%$ of the range of the parameters. To avoid a blockage of the solution evolution, the size of the restrained domain is increased by $4 \%$ each time no better solution can be found. If the search space increase reaches $40 \%$, the second step is stopped. To rank the solutions, each mechanism is tested and associated to its performance value. Non-viable mechanisms are associated with a null value. It is important to note that the algorithm is quite robust with the thresholds used to restrain or widen the search space. Their values are easily set and do not strongly affect the success of the optimization.

In the third step, solution generation is performed by switching to a gaussian distribution law for the evolution of each parameter. The mean value and standard deviation are 


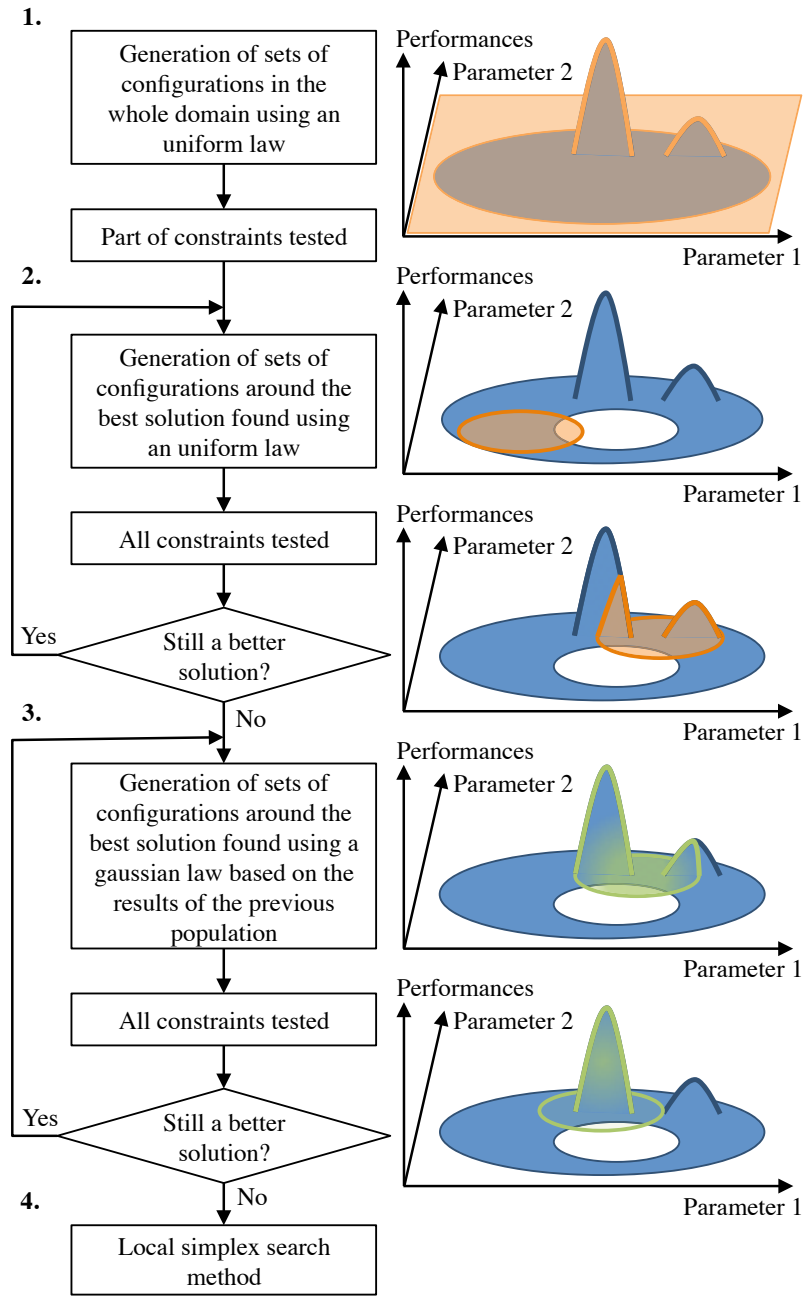

Figure 10. Algorithm structure with a simplified illustration of a two parameters problem for each step.

computed using the sets of viable configurations determined in the second step.

The standard deviation of each parameter tends to zero during the third step. When all the values become small enough, local search using a simplex search method is performed to refine the solution.

\subsection{Optimization of the active stabilizer}

Among possible piezoelectric actuators, a device from PI, $\mathrm{P}-007.40$, is considered. It has one of the largest available displacements $(60 \mu \mathrm{m})$ with a diameter of $7 \mathrm{~mm}$ which allows its integration. Its length is equal to $50 \mathrm{~mm}$, and the maximal force is $1150 \mathrm{~N}$. A $12 \mathrm{~mm}$ diameter shaft in $35 \mathrm{NCD} 16$ steel is considered.

The ACO is implemented using the Matlab software. Even though such an implementation is not optimal, optimization for our problem was achieved in less than six hours.

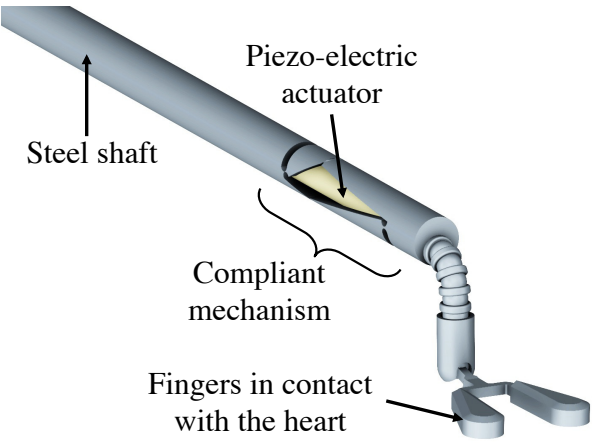

Figure 11. Cardiac stabilizer and its integrated compensation compliant mechanism.

With the optimal solution (Fig. 11), the stabilizer can exhibit a displacement of $0.9 \mathrm{~mm}$, with maximal stress of $550 \mathrm{MPa}$. This latter value corresponds to the endurance limit of the material. It is important to note that the performance of the device is confirmed by comparison with FEA results, performed using PTC Pro/Mechanica. Relative errors in the estimation of the displacement and the stresses in the compliant joints do not exceed $5 \%$. The PRBM describes accurately the behavior of the device, and combining ACO with PRBM allows us to introduce an active stabilizer whose performance corresponds to the medical requirement.

\subsection{Experimentation}

Interesting results have been obtained in terms of mechanism optimization and design of the medical device. Experimentation has been carried out to confirm these results. For availability reasons, a slightly different piezoelectric actuator is chosen: the P-041.30 has a diameter of $12 \mathrm{~mm}$, length of $80 \mathrm{~mm}$ and can provide a maximum displacement of $45 \mu \mathrm{m}$. A Z30C13 stainless steel tube of $18 \mathrm{~mm}$ diameter is chosen. The yield limit of the material is $500 \mathrm{MPa}$.

Optimization is performed using these new specifications. The results of the ACO are again in very good accordance with the FEA. The discrepancy in the estimation of the displacement $d$ is below $4 \%$ and below $7 \%$ for the stresses. The device is designed to exhibit a maximal displacement of $0.5 \mathrm{~mm}$.

The prototype is manufactured using $\mathrm{CNC}$ machining (Fig. 12). Its performance is evaluated using a visual marker located at the tip of the prototype and a high speed camera that allows the determination of the marker displacement. The maximum displacement is estimated as $0.5 \mathrm{~mm}$ (Fig. 13), the value obtained with the PRBM model as well as the FEA. Loads have been applied on the stabilizer tip to evaluate the consistency of PRBM model, FEA and experimental results. For a load up to $5.7 \mathrm{~N}$, above the target value of $5 \mathrm{~N}$, relative errors between the three displacement values are below $5 \%$. Finally an analysis of the first eigenfrequency 


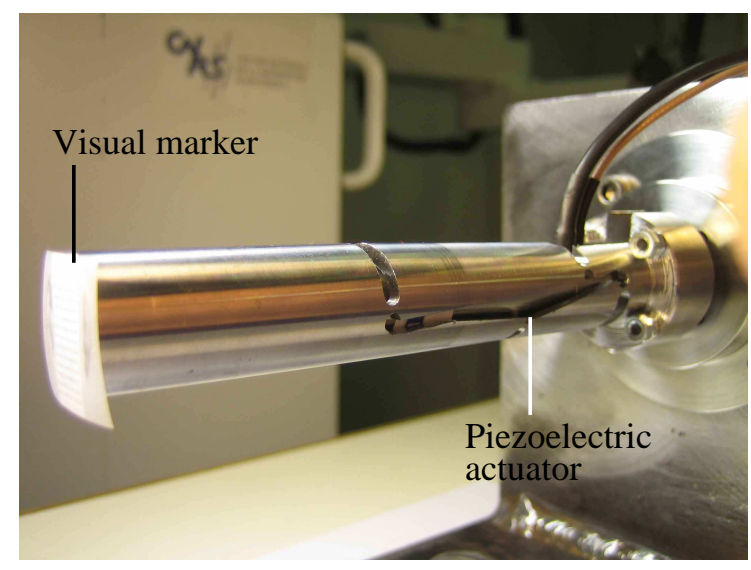

Figure 12. Prototype assembly and its machined compliant mechanism.

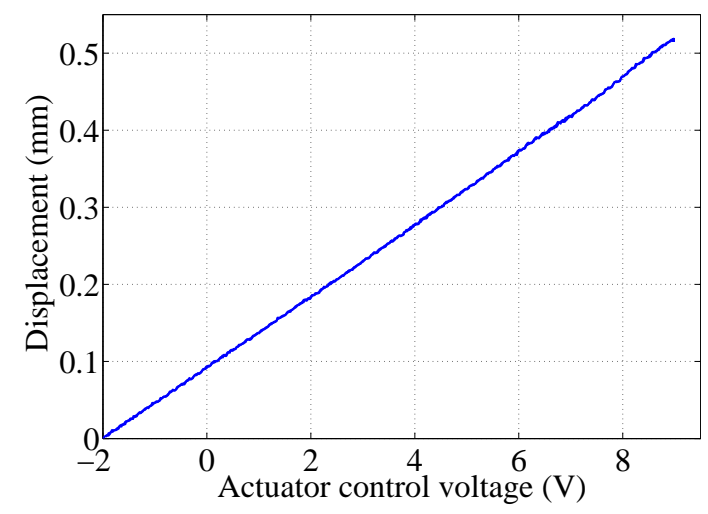

Figure 13. Experimental measure of the displacement.

has been performed. Its value is around $200 \mathrm{~Hz}$, with $10 \mathrm{~Hz}$ of measurement uncertainty, whereas FEA simulation provides a value of $203 \mathrm{~Hz}$.

Experimental results are very close to those obtained with the numerical simulation and the PRBM. This tends to show that the proposed modeling reliably describes the behavior of the device. As a consequence, the performance of the device with the $12 \mathrm{~mm}$ diameter tube, compatible with the medical application, should be experimentally achievable.

\section{Conclusions}

In this paper, the use of compliant mechanisms in the context of the design of a new surgical device has been presented. First, we have shown that combining compliant mechanisms with piezoelectric actuation can allow the development of a new surgical device. Then, in a second step, we have outlined that the surgical requirements can lead to the development of original compliant architectures. A remote center of motion architecture has been developed based on original mechanical amplifiers. The development of active stabilizers is now related to the development of highly integrated compliant architectures, and their associated design methodologies. We have proposed the use of Ant Colony Optimization, which gives interesting results according to the first numerical and experimental evaluation of our proof-of-concept.

From a medical point of view, active stabilization is a promising approach, and current results on a fully integrated stabilizer open new perspectives. The application area of such a mechanism could actually be widened, for instance, to tremor compensation in microsurgery or micropositioning.

From a compliant mechanism design point of view, using Ant Colony Optimization for the optimization of PRBM of compliant mechanisms may constitute a new approach for the synthesis of compliant structures. Further work in the design of mechanisms such as parallel architecture for multi-DOF systems will now be carried out.

Edited by: A. Barari

Reviewed by: two anonymous referees

\section{References}

Awtar, S., Trutna, T., Nielsen, J., Abani, R., and Geiger, J.: FlexDex: A Minimally Invasive Surgical Tool with Enhanced Dexterity and Intuitive Actuation, ASME Journal of Medical Devices, 4, 8 pp., 2010.

Bachta, W., Renaud, P., Laroche, E., Forgione, A., and Gangloff, J.: Design and control of a new active cardiac stabilizer, IEEE/RSJ Int. Conf. on Intelligent Robotos and Systems - IROS, 404-409, 2007.

Bachta, W., Renaud, P., Laroche, E., Forgione, A., and Gangloff, J.: Cardiolock: an active cardiac stabilizer, first in vivo experiments using a new robotized device, Comput. Aided Surg., 13, 243 254, 2008.

Bachta, W., Renaud, P., Laroche, E., and Gangloff, J.: Cardiolock2: Parallel singularities for the design of an active heart stabilizer, in: ICRA, 3839-3844, 2009.

Blum, C.: Ant colony optimization: Introduction and recent trends, Phys. Life Rev., 2, 353-373, 2005.

Cattin, P., Dave, H., Grunenfelder, J., Szekely, G., Turina, M., and Zund, G.: Trajectory of coronary motion and its significance in robotic motion cancellation, Eur. J. Cardio-Thorac., 25, 786790, 2004.

Choi, K.-B., Lee, J. J., and Hata, S.: A piezo-driven compliant stage with double mechanical amplification mechanisms arranged in parallel, Sensor. Actuat. A-Phys., 161, 173-181, 2010.

Dorigo, M. and Gambardella, L. M.: Ant colonies for the travelling salesman problem, Biosystems, 43, 73-81, 1997.

Frecker, M. I., Powell, K. M., and Haluck, R.: Design of a Multifunctional Compliant Instrument for Minimally Invasive Surgery, J. Biomech. Eng., 127, 990-993, 2005a.

Frecker, M. I., Schadler, J., Haluck, R. S., Culkar, K., and Dziedzic, R.: Laparoscopic Multifunctional Instruments: Design and Testing of Initial Prototypes, JSLS, J. S. Laparoend., 9, 105-112, 2005b. 
Gosselin, C. and Angeles, J.: The optimum kinematic design of a spherical three-degree-of-freedom parallel manipulator, J. Mech. Transm.-T., 111, 202-207, 1989.

Gosselin, C. and Angeles, J.: Singularity analysis of closed-loop kinematic chains, IEEE T. Robotic. Autom., 6, 281-290, 1990.

Gregorio, R. D.: Kinematics of the 3-RSR wrist, IEEE T. Robotic. Autom., 20, 750-754, 2004.

Howell, L.: Compliant mechanisms, Wiley-IEEE, 2001

Kaveh, A., Hassani, B., Shojaee, S., and Tavakkoli, S.: Structural topology optimization using ant colony methodology, Eng. Struct., 30, 2559-2565, 2008.

Lemma, M., Mangini, A., Redaelli, A., and Acocella, F.: Do cardiac stabilizers really stabilize? Experimental quantitative analysis of mechanical stabilization, Interactive Cardiovascular and Thoracic Surgery, 4, 222-226, 2005.

Liaw, H. C. and Shirinzadeh, B.: Robust generalised impedance control of piezo-actuated flexure-based four-bar mechanisms for micro/nano manipulation, Sensor. Actuat. A-Phys., 148, 443453, 2008.

Luh, G.-C. and Lin, C.-Y.: Structural topology optimization using ant colony optimization algorithm, Appl. Soft Comput., 9, 13431353, 2009.

Parkinson, M. B., Jensen, B. D., and Kurabayashi, K.: Design of compliant force and displacement amplification micromechanisms, Proceedings of ASME Design Engineering Technical Conferences, DET2001/DAC-21089, 2001.

Pham, H. and Chen, I.: Workspace and Static Analyses of 2-DOF Flexure Parallel Mechanism, in: Int. Conf. on Control, Automation, Robotics and Vision, 968-973, 2002.

Pilkey, W. D. and Pilkey, D. F.: Peterson's Stress Concentration Factors, 3rd Edn., John Wiley \& Sons, 2008.
Ranganath, R., Nair, P. S., Mruthyunjaya, T. S., and Ghosal, A.: A force-torque sensor based on a Stewart Platform in a nearsingular configuration, Mech. Mach. Theory, 39, 971-998, 2006.

Rebello, K.: Applications of MEMS in surgery, Proc. IEEE, 92, 43-55, 2004.

Schotborgh, W. O., Kokkeler, F. G. M., Tragter, H., and van Houten, F. J. A. M.: Dimensionless design graphs for flexure elements and a comparison between three flexure elements, Precis. Eng., 29, 41-47, 2005.

Sitti, M.: Piezoelectrically actuated four-bar mechanism with two flexible links for micromechanical flying insect thorax, IEEEASME T. Mech., 8, 26-36, 2003.

Socha, K. and Dorigo, M.: Ant colony optimization for continuous domains, Eur. J. Oper. Res., 185, 1155-1173, 2008.

Stoughton, R. and Arai, T.: Kinematic optimization of a chopstickstype micromanipulator, in: Japan-USA Symp. on Flexible Autom., USA, 4472-4477, 1992.

Tian, Y., Shirinzadeh, B., Zhang, D., and Alici, G.: Development and dynamic modelling of a flexure-based Scott-Russell mechanism for nano-manipulation, Mech. Syst. Signal Pr., 23, 957978, 2009.

Trease, B., Moon, Y.-M., and Kota, S.: Design of LargeDisplacement Compliant Joints, J. Mech. Design, 127, 788-798, 2004.

Yong, Y. K., Lu, T.-F., and Handley, D. C.: Review of circular flexure hinge design equations and derivation of empirical formulations, Precis. Eng., 32, 63-70, 2008. 